vast amount of information and many interesting contacts. It also helped develop working concepts for the simulated MCI. It is the first of many steps that must be taken to become prepared for a possible future MCI.

Keywords: education; mass casualty; preparedness; simulation; training; workshops

Prebosp Disast Metl 2005;20(2):554-s55

\section{Disaster Training Competencies for Healthcare Workers}

E.B. Hsu; ${ }^{1}$ T.L. Thomas, ${ }^{2}$ D.M. Whyne, ${ }^{1}$ E.B. Bass; ${ }^{3}$

G.D. Kelen; ${ }^{1}$ G.B. Green ${ }^{1}$

1. Johns Hopkins Office of Critical Event Preparedness and Response (CEPAR), Baltimore, Maryland USA

2. Loma Linda University, Loma Linda, California USA

3. Johns Hopkins Evidence-Based Practice Center, Baltimore, Maryland USA

Introduction: Although training and education have long been accepted as an integral part of disaster preparedness, many current practices have not been developed using evidence-based methods. The need for effective training of healthcare staff at all levels and "development of standards and guidelines for education and training in the multi-disciplinary health response to major events" has been identified by the 13th World Congress on Disaster and Emergency Medicine (Melbourne) as one of its highest priorities. Systematic evidence-based methods were described to derive competencies relevant to all hospital healthcare workers and staff during disasters.

Methods: A competency was defined as a broad area of personal capability, including knowledge and skill, and the application of that knowledge and skill to the standard of performance required. Cross-cutting competencies were intended to be applicable to all hospital, healthcare workers and staff. The conceptual development of competencies incorporated an evidence-based process with the following steps: (1) systematic review of existing competencies, courses, and training objectives, peer-reviewed literature, and educational theory; (2) synthesis of new competencies; (3) expert panel review; (4) refinement of new competencies; and (5) development of terminal and enabling objectives for each competency covering requisite knowledge, attitudes, and skills. Results: Through a structured, iterative, evidence-based process, the following seven competencies were developed: (1) recognize a potentially critical event and implement initial actions; (2) apply the principles of critical event management; (3) demonstrate critical event safety principles; (4) understand the institutional emergency operations plan; (5) demonstrate effective critical event communications; (6) understand the incident command system and your role in it; and (7) demonstrate the knowledge and skills needed to fulfill your role during a critical event. For each of the cross-cutting competencies, comprehensive terminal and enabling objectives were developed.

Conclusion: Cross-cutting competencies developed through a systematic evidence-based approach serve as a paradigm for hospital, healthcare worker disaster training and education. Keywords: competencies; disaster; education; evidence-base; healthcare; hospital; incident command system; management; objectives; recognition; safety; training; workers

Prehosp Disast Med 2005;20(2):s55

\section{Free Papers Theme 19: Infectious Disease}

\author{
Attitudes, Concerns, and Knowledge of Taiwanese \\ Medical Laboratory Technologists and Students \\ Regarding HIV and AIDS \\ F.F.S. Wu, ${ }^{1}$ R. Siebers, ${ }^{2}$ J.H. Liu, ${ }^{3}$ C.Y. Horng, ${ }^{3}$ \\ Y.P. Huang, ${ }^{3}$ J.Y. Lin, ${ }^{3}$ J.Y. Tsay, ${ }^{3}$ M. J. Gu, ${ }^{3}$ \\ M.H. Huang, ${ }^{3}$ C.W. Juan ${ }^{3}$ \\ 1. Changhua Show Chwan Memorial Hospital, Taiwan \\ 2. Wellington School of Medicine and Health Sciences, New \\ Zealand \\ 3. Show Chwan Memorial Hospital, Taiwan
}

Background: The World Health Organization (WHO) estimated that in 2003 there were almost 38 million people living with Human Immunodeficiency Virus (HIV) worldwide. Medical laboratory workers are the second largest professional group with potential exposure to HIV through handling of biological specimens. Previous studies from the United States, New Zealand, the South Pacific, and Finland have shown that medical laboratory workers have many unfounded fears regarding HIV and Acquired Immune Deficiency Syndrome (AIDS) that impact their work practices and attitudes towards AIDS patients.

Objective: To describe the practices and concerns of medical laboratory technologists and medical laboratory technology students regarding HIV/AIDS in central and southern Taiwan.

Methods: Questionnaires were distributed to all medical laboratory technologists and medical laboratory technology students in four hospitals (Pingtung Christian Hospital, Show Chwan Memorial Hospital, Changhua Christian Hospital, Zhushan Show Chwan Hospital) and three medical laboratory technology schools (Chunghwa Medical Technology College, Chungtai Medical Technology College, Chung Shan Medical University) in central and southern Taiwan.

Results: Of a potential pool of 530 persons, 380 (144 students and 236 medical laboratory technologists) returned questionnaires (response rate $=72 \%$ ). There was no significant difference in response rates between students and medical laboratory technologists. Respondents were predominantly female (77\%) and $196(52 \%)$ previously had attended workshops or lectures specifically on HIV/AIDS.

More than ninety percent of the respondents always wore gloves when handling a variety of biological specimens and $63 \%$ treated all specimens as potentially HIVpositive. There was a significant association between the use of gloves and the concern about acquiring HIV/AIDS or hepatitis at work $(p<0.001)$. Respondents were concerned similarly about acquiring HIV or hepatitis $B$ in their work place ( $84 \%$ and $77 \%$, respectively). A total of 44 medical laboratory technologists were seriously considering leaving the workforce because of potential contact with AIDS patients or HIV-positive biological samples. This was, in turn, related to their training and practical HIV/AIDS knowledge. 